\title{
Free Space Measurement for Blind Person using Histogram Equalization and Adaptive Region Growing
}

\author{
Hari Raksha K Malali, Nithin Kashyap, Natesh M
}

\begin{abstract}
Although significant research has been conducted to improve the standard of life of the blind person by using recent methods, it continues to be adequate for blindness rehabilitation. Increasingly, the computer vision system is being used to improve the performance of blind life's. In this paper, a technique is designed using histogram equalization and adaptive region growing to aid the blind person by giving the person information about the free space apart from the obstacles around him in all the directions for better mobility. It encompasses three modules, (i) histogram equalization (ii) segmentation and (iii) Kalman filtering. Histogram equalization module employs a canny edge detector to detect edges and then goes through with histogram equalization. Segmentation module makes use of an adaptive region growing for the segmentation process. Kalman filtering and comparison is made use of the final module to calculate the free space available. The input image is Kalman filtered and compared with the segmented image to have the free space calculation. The comparison is carried out with the help of the OR operator and the resulting figure give the free space. The proposed strategy is assessed under standard assessment measurements of False Positive, False Negative, True Positive and True Negative, explicitness, affectability, and precision for various group sizes. The simulations results obtained are plotted. This resulted in the following observations highest specificity, sensitivity and accuracy came around 0.90, 0.50 and 0.71 and similarly, average TP, TN, $F P$ and $F N$ came about $0.79,0.5,0.5$ and 0.20 respectively. The high evaluation metric values indicate the good performance of the proposed technique in the area.
\end{abstract}

Keywords: Free Space Measurement, Adaptive Region Growing, Kalman Filter, Canny Edge Detector, Histogram Equalization Segmentation, Floor detection.

\section{INTRODUCTION}

Power of seeing is great significance and eyes play a vital role. Vision empowers people to accomplish tasks and to learn about the world around them. Impairment caused because of many reasons like sightlessness by birth, numerous damages to the brain. People who suffer from blindness are affected by visual impairment, face difficulties in movement apart from many other problems. This condition leads the concerned person to be handicapped and needs guidance or assistance for every action [1, 2]. Due to this, there are many difficulties for purblind and blind people

Revised Version Manuscript Received on 10 September, 2019.

HariRaksha K Malali, Department of Computer Science and EgineeringVidyavardhaka College of Engineering, Mysuru, Karnataka, India

NithinKashyap, Department of Computer Science and EgineeringVidyavardhaka College of Engineering, Mysuru, Karnataka, India

Natesh M, Department of Computer Science and EgineeringVidyavardhaka College of Engineering, Mysuru, Karnataka, India in society, as they have to encounter any type of hurdles in accomplishing everyday routine works [3]. In order to help these visually impaired people, advancements day by day in the area of science introduces new technologies and rehabilitations to make their life easy and comfortable. Over the decades, these peoples were using the methods of pathfinding aids like guide dogs, white cane, etc. The long white cane is an age-old transportability appliance which is used to give blind people the strength to triumph and to enjoy their independent life by eventually detecting obstacles in the path for them. On the other hand, training dogs did help them to guide their paths by detecting hindrances around. With the growing science and technologies today we have much modern equipment's to assist and guide the blind. They are generally known as Electronic Travel Aids. Walking safe and sound and self-confidently without any human aid in built-up or unknown environments is a herculean task for blind people [2]. But still, the developed instruments aren't satisfactory for the rehabilitation of visual defect [1].

Many scholars have proposed devices for improving these visually impaired people life quality. Human vision abilities are extraordinary to realized images with the imbibed images in the brain, but these are also having some limitations like being tired, slow, and not so accurate because of some disease $[1,4]$. These limitations may be rectified by using the principles of computer vision system which definitely improves the blind life quality. Few researchers have proposed outdoor navigation devices for vision affected person [1]. The aim of computer vision is to create computers "see" and seeing refers understanding scenes, reconstructing 3D objects, recognizing objects avoiding obstacles serving to the visually impaired folks by processing pictures or video using machine learning, sense of pure mathematics and applied mathematics. By understanding such things as how images are shaped, data about the sensors (cameras), and data about the real world, it is possible to surmise data about the world from an image or set of images [5, 6]. For instance, individual may desire to know the shade of an orange, the thickness of a printed circuit, the dimensions of an obstacle in front of a robot on Earth, the uniqueness of a person's face in a face recognition system, the motion of an entity, the vegetation type of the ground below, or density measurement of cartilage tissue - automatically, from images. The Computer vision analyses how proficiently and robustly can

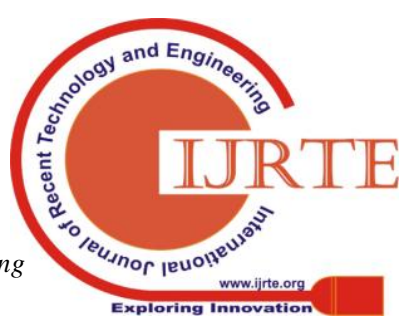


a given mission be done. Computer vision is a sub-domain of the 21 st century's engineer's favorite domain artificial intelligence; and also this has been a dynamic area of research for almost four decades now. Every day from depositing checks, use of the license plate reader technology to alerting humans to prevent mishaps and maintenance, the role of computer vision is endless [7].

The goal of computer vision is to change computers see the world. By employing a camera because the eye of a computer, studies in computer vision request to develop higher suggests that to capture and extract helpful visual data from pictures and videos and to use such data to mechanically interpret the gorgeous world encompassing us. Applications vary from errands like modern machine vision frameworks that, state, look at jugs dashing by on a mechanical framework, to the investigation into registering and computers or robots that may grasp the Earth around them. The machine vision and computer vision fields have indispensable cover $[5,8]$. Computer vision covers the center real innovation of machine-driven picture investigation that is utilized in different fields. Machine vision ordinarily alludes to a procedure of blending machine-driven picture investigation with elective courses and advances to create golem steerage and machine-driven examination in mechanical applications. As a subject, computer vision cares with the thought behind counterfeit frameworks that concentrate information from pictures [9].

As an innovative subject, computer vision plans to utilize its models and speculations for the improvement of computer vision frameworks. Tests of utilizations of computer vision grasp frameworks for, prevailing procedures, Navigation that is by Associate in Nursing self-sufficient vehicle or portable machine, analyst work occasions, organizing data, Modelling articles or situations (e.g., Geographic's displaying or Medical picture examination) and Interaction. Sub-areas of computer vision grasp scene reproduction, video following, occasion location, visual recognition, ordering, learning, picture rebuilding, and movement estimation. In the vast majority of the computer vision applications, computers are unit pre-customized to disentangle a specific assignment, anyway techniques upheld learning region unit as of now transforming into dynamically regular [6,7].

Image-based floor detection [10] is an application of laptop vision and it may be incorporated for navigation of blind individuals. Here the navigation relies on finding the free house occupancy. By having an idea where the ground is, the visually impaired person can evade obstacles by navigating inside the house with detection of the ground, the visually handicapped person is also ready to get the data that will help in coming up with a map of the surroundings, to that degree because the floor detection delineates between the ground and also the wall. In addition, restriction exploitation A current guide might be radio-constrained by floor identification by coordinating the situation of the distinguished floor with the position of the ground anticipated from the guide additional purposes behind floor discovery [10] grasp issues like processing the elements of the zone. A noteworthy amount of investigation has focused upon the snag evading drawback. In these procedures, the main reason for existing is to see the free house on the double round the outwardly crippled individual rather than the specific divider floor limit. The greater part of those methodologies utilizes the base plane confinement supposition to live whether the disparity or movement of pixels coordinates the qualities that will be normal if the focuses are on the base plane.

In this paper, a technique is designed to aid the blind person using edge detection. The technique aims to give the person information about the free space apart from the obstacles around him in all the directions for better mobility. The technique comprises of three segments, namely histogram equalization module, segmentation module, and Kalman filtering module. In the histogram equalization module, canny edge detector is employed to detect edges and subsequently histogram equalization is carried out. Subsequently, the adaptive region growing is employed in a segmentation module to complete the segmentation process. In Kalman filtering module, the input image is Kalman filtered and compared with the segmented image to have the free space calculation. The comparison is carried out with the help of the OR operator and the resulting figure give the free space.

The remainder of the paper is composed as pursues: Section 2 refers to the literature review. Section 3 offers the inspiration and section 4 depicts the anticipated strategy. Section 5 offers results and discussion. The end is summed up in Section 6.

\section{LITERATURE REVIEW}

There have been several works associated with visually handicapped person navigations help. Dhanshri and Kashwan [1] aimed towards finding a viable and easy resolution for near-blind persons at half value. This paper was a trial to report a comprehensive methodology to style, characterize and check physical science system for the help of blind persons. The simulation checks were dispensed exploitation MATLAB and pictures of test objects were noninheritable on-line by exploitation NI-Lab VIEW platform. The most objectives were to amass a picture of an obstacle, determine it, live a distance from current location and eventually convert text into synthesized. João José et al. [2] designed and conferred a Smart Vision image. It had been a little, minimal effort and essentially wearable route help for visually impaired and outwardly debilitated people. Its common sense tends to world route for controlling the client to some fate, and a local route for arranging techniques, walkways, and halls, with dismissing of static also as moving hindrances. Local route applies to each all through entryways things. They focused on the local route: the identification of way fringes and impediments before of the client and basically on the far side the scope of the white stick, such the client are regularly control helped with centering on the trail and cautioned to approaching dangers. utilizing a stereo camera worn at chest stature, a transportable computer in a very shoulder-lashed pocket or pocket and just a single earpiece or little speaker, the framework was subtle, it had been no prevention though strolling with the stick, and it doesn't square conventional encompass sounds. The vision

Blue Eyes Intelligence Engineering

\& Sciences Publication 
calculations were advanced such the framework will take a shot at certain casings every second.

Nithya and Shravani [3] given an electronic travel help for visually impaired people to explore securely and rapidly, a snag detection system victimization UVC camera-based for the most part visual route has been thought of. The anticipated framework identified the deterrents up to $300 \mathrm{~cm}$ by means of estimating framework and sends input in kind of signal sound to tell the individual in regards to its area. Additionally, to the present, UVC digital camera was connected to 32-bit ARM small controller that supports options and algorithms for coming up with of blind individual's steerage stick. This supported image process that was wont to processes pictures and provides voice response when detection that was used for locating the properties of the obstacle especially, within the context of the work. Identification of human presence was supported by face detection and object detection. The algorithms were enforced in open $\mathrm{CV}$, which runs on Linux setting. Lorenzo Picinali et al. [4] examined the odds of supporting visually impaired individuals in learning a deliberation setting setup through the tuning in of sound occasions and their associations with these occasions among a computer game expertise. A correlation of 2 styles of learning through methodology investigation was performed: set up genuine dislodging and dynamic route during a virtual plan. The virtual route rendered exclusively acoustic information. Results for two groups of five members demonstrated that intelligent investigation of virtual acoustic territory reproductions will offer agreeable information for the advancement of rational reflection mental maps, however, a few varieties were found between the 2 environments tried inside the trials. Additionally, the substance of the about explored situations protected topological and metric properties, as was found through a genuine route.

BhuvaneshArasu and SenthilKumaran [11] anticipated a device to help an outwardly hindered individual to carry on with an actual existence like some other individual on this world with no close to the home guide. This device utilized the idea of echolocation utilized by bats. Numerous parts were used in building up this gadget in this manner known as the visually impaired man's eye like supersonic sound producer ultrasonic sound collector, micro-controller, camera, and a steel pole. To detect the elements of the thing, the force of returned supersonic wave was utilized. Doppler's impact decided the articles speed, bearing and movement (towards or away) of the thing chose. Making utilization of point recognition procedure it decided whether the individual was strolling attempting down or keeping his head straight. Dijkstra's calculation was fundamentally used to help locate the most limited way in order to control the outwardly disabled client from the present way. The Doppler's impact gave an effect was to look out the overall movement of the items, towards or a long way from the client. Vincent Gaudissar [12] depict an implanted gadget committed for visually impaired people. The most point of the framework was to make a programmed content perusing right-hand misuse existing equipment identified with creative calculations. A private advanced aide (PDA) was picked because of it joined little size, machine assets, and low worth. 3 key innovations were fundamental: content location, optical character acknowledgment, and discourse union. In addition, to be as conservative as achievable, a chose interface was made to answer blind people demands.

H. Fernandes et al. [13] displayed a stage in order to give and deal with geographic data, including accessibility oriented features. This geographic data framework otherwise called GIS was a piece of a greater task, which was named Smart Vision. The desired outcome of this venture was to make a framework which enabled visually hindered clients to move around in the University of Alto Douro and Trás-os-Montes grounds. This GIS stage, all in all with different segments of the Smart Vision framework model, gave information to outwardly disabled clients, managing their bearing finding and offering admonitions to the close impediments or focal points. Together with the GIS stage, the paper likewise portrayed the treatment of openness data by the Smart Vision model, in particular, the Navigation Module, the Computer Vision Module, and the Interface Module. Anke M. Brock et al. [14] showed a relationship of the comfort of a built-up raised-line map versus a wise guide reached the screen, a raised-line overlay, and sound yield. The two maps were attempted by 24 visually hindered individuals. They checked convenience as profitability, sufficiency, and satisfaction. Our results showed that replacing braille with fundamental sound material coordinated effort basically improved capability and customer satisfaction. Capability was not related to the guide type yet rather rely upon client's properties similarly as the classification of evaluated spatial learning. Whole deal assessment of acquired spatial data revealed that maps, paying little heed to whether instinctive or not, were useful to build amazing audit type mental depictions in outwardly debilitated. Outright, these outcomes were promising as they show that instinctive maps were a tolerable response for improving aide examination and scholarly mapping in outwardly incapacitated people.

S. Kammoun et al. [15] proposed the NAVIG venture which secured significant disadvantage factors: (1) situating precision given by these gadgets was not adequate to manage a VI person on foot, (2) frameworks depended on Geographical Information Systems not adjusted to walker versatility, and (3) the direction techniques ought to be adjusted to the errand of passer-by route. The NAVIG venture meant to answer every one of these impediments through a participatory structure system with the VI and orientation and versatile teachers. The NAVIG gadget expected to supplement regular ways (for example white stick and guide hound), while likewise adding interesting features to restrict explicit items in the earth, re-establish some visuomotor capacities, and help route.Wersényi, György et al. [16] displayed a virtual localization for visually impaired people. All together for visually impaired individuals to all the more likely utilize COMPUTERs, a sound-related virtual condition was utilized to display data that may somehow or another be accessible just with vision. Sound-related items can be spatial put in the virtual condition if the client can effectively recognize their area. As opposed
Blue Eyes Intelligence Engineering \& Sciences Publication 
to located subjects, blind subjects were better at identifying movements in the flat plane around the head, localizing static frontal sound sources, and orientation in a 2-D virtual sound presentation. Then again, sighted subjects performed better recognizing rising sound sources in the vertical plane and distinguishing static sources in the back.

\section{MOTIVATION}

Vision plays a crucial role in human sensitivity about the surrounding atmosphere and mobility is one of the biggest hindrances faced by a blind person in their day-to-day life. Many researchers have proposed devices for improving the blind person's life quality. Many researchers have proposed devices for improving the blind person's life quality. Human vision abilities are extraordinary to realized images with the imbibed images in the brain, but these are also having some limitations like being tired, slow, and not so accurate because of some disease. These limitations may be rectified by using the principles of computer vision system which definitely improves the blind life quality. Image-based floor detection is an application of computer vision and it can be incorporated for navigation of blind people. Depth estimation in 2-D images is a difficult task and to do this one has to really estimate the depth of individual objects and magnitude of all techniques.

\section{FREE SPACE MEASUREMENT FOR BLIND PERSON USING HISTOGRAM EQUALIZATION AND ADAPTIVE REGION GROWING}

The proposed strategy is intended to help the visually impaired individual by giving the individual, data about the free space separated from the obstacles around him in every direction for better portability. The strategy utilizes histogram equalization and versatile region developing. The method involves three modules, specifically histogram equalization module, segmentation module, and Kalman filtering module. The square chart of the proposed system is given in figure 1.

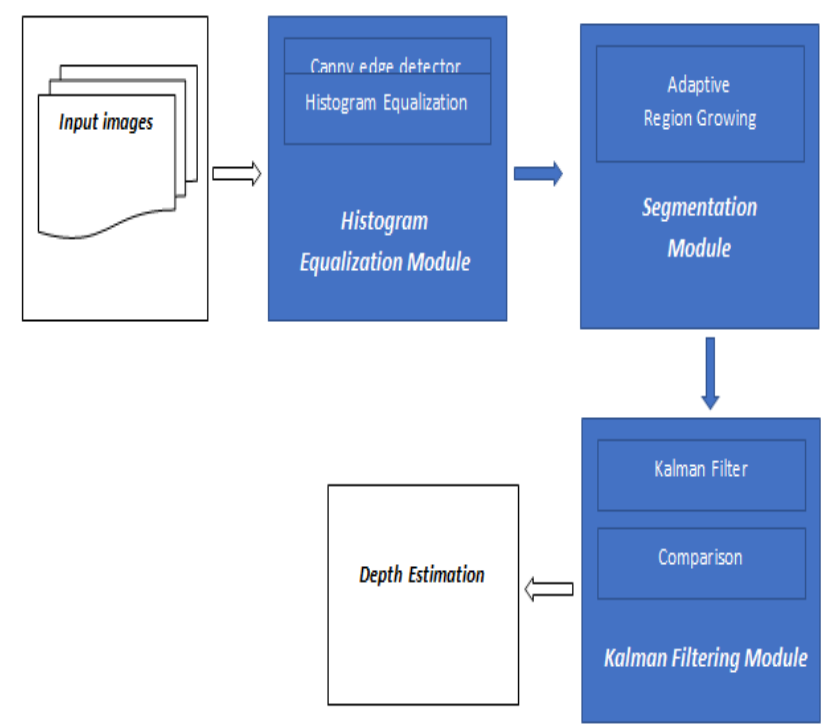

Figure 1: Block diagram using histogram equalization and region growing technique the images has to be computed by applying different

\subsection{Histogram Equalization Module}

The initial step of the methodology observes force edges by applying the shrewd edge detection [17] to utilize histogram equalization. Edges are essential native changes of intensity in an image. Edges by and large happen on the limit between two entirely unexpected areas in an image. Edge detection goes for recognizing points in a computerized picture at that the picture brightness changes strongly or, a great deal of formally, has discontinuities that are commonly sorted out into a gathering of curvilinear line sections named edges. In our strategy, we will, in general, utilize canny edge finder. The canny edge watches a position identification administrator that uses a multi-organize algorithmic program to distinguish an enormous scope of edges in pictures. Canny edge detector has the upside of getting low blunder rate.

For canny edge detection, at the start, the image is born-again to greyscale image and therefore the following processes [18] are carried out:

- Smoothing

- Finding gradients

- Non-maximum suppression

- Double thresh holding

- Edge tracking by hysteresis

The steps involved and its output for a sample image is given in figure 2 .

Input Image

Smoothing and Gradient

Non- extreme

dominance

Double thresh holding and Edge tracking by hysteresis

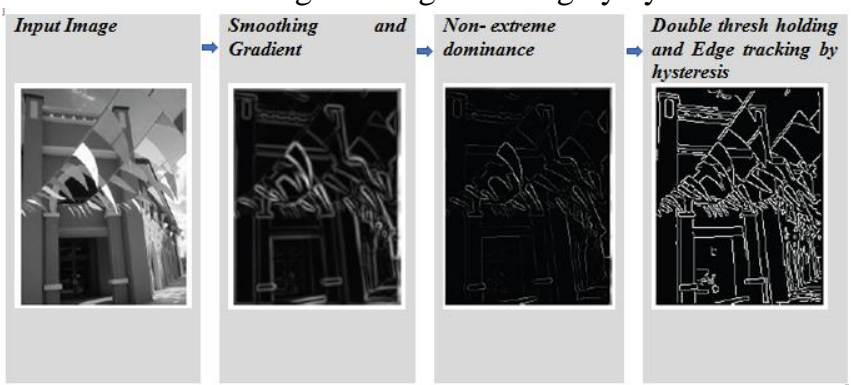

Figure 2: Steps for sample image using Canny edge method

\section{a) Smoothing}

The smoothing process is a compound blur of the image by the Gaussian filter used to remove

sound by default all pictures captured by a camera contain a certain degree of noise.

In order to prevent the sound that is mistaken for the edges, it should be reduced. Therefore

in order to reduce noise, the first Gaussian filter is applied to the image of the sanding process.

Consider an image can be represented by $\mathrm{I}(\mathrm{p}, \mathrm{q}), \mathrm{G}(\mathrm{p}, \mathrm{q})$ the Gaussian filter, and then the

smoothed resulting image $S(p, q)$ It can be represented by $\mathrm{S}(\mathrm{p}, \mathrm{q}) \square \mathrm{G}(\mathrm{p}, \mathrm{q}) \square \mathrm{I}(\mathrm{p}, \mathrm{q})(1)$ 


\section{b) Gradient}

The main objective is to score the edges, where the change of direction of the intensity orcolor in an image that has larger sizes. There is an algorithm called the Canny algorithm thattries essentially the edges where the range of monochrome shades from black to whitechanging image. These places are located by determining the image gradients. Gradientimage for each pixel in the smoothed image can be determined by applying a so-called Sobeloperator. Thereafter, image slope must be approached in both the $\mathrm{x}$ and $\mathrm{y}$ directions,respectively, from the nuclei image gradient amplitudes ( $R$ ) Can then be found as athe measure of Euclidean distance by applying the law of Pythagoras, which is given by:

\section{| R | $\square$ RX $2 \square$ RY 2 (2)}

$\mathrm{RX}$ and RY They are respectively image gradients in the $\mathrm{x}$ and $\mathrm{y}$ directions.

\section{c) Non-maximum suppression}

The significance of this stage is by turning the blurry edges of the gradient magnitude to achieve sharp edges in the image. This is accomplished basically by keeping all local maxima in the gradient of the image and eliminating everything else. First-round the image gradient path close 45o. Next, match the present pixel border intensity acquired by the strength of the border cells in both favorable and adverse orientation of the gradient. If the strength collected from the present pixel border is higher, the significance of the marginal strength will be preserved. If the valuation is not higher, delete it.

\section{d) Double thresholding}

Seeing the perspective boundaries guided by thresholding is controlled. The surviving edge-pixels are laid apart with their value pixel-by-pixel when the most severe concealment phase is (yet) laid apart. Some of these may in all likelihood be real corners inside the image, but some are also carried about by clamor or shading variants due to rough textures, for example. The most perfect strategy to determine the use of an angle between these futures, so solely surfaces more rooted that an accurate value would be protected. The algorithmic program for shrewd border detection utilizes double thresholding. Edge pixels more grounded than the high edge are set as solid; edge pixels are stifled flimsier than the low edge and edge pixels are set as powerless between the two edges.

\section{e) Edge tracking by hysteresis}

Here, final edges are determined by suppressing all edges that don't seem to be connected to a really robust edge. Robust edges are taken as "certain edges", and may right away be enclosed within the final edge image. Weak edges are enclosed if and given that they're connected to robust edges. The logic is in fact that noise and different little variations are unlikely to lead to a powerful edge. Therefore, robust edges would solely flow from true edges within the original image. The weak edges will either flow from to true edges or noise/color variations. The latter kind can most likely be distributed severally of edges on the complete image, and therefore solely a tiny low quantity is set adjacent to robust edges. Weak edges due to true edges are rather more possible to be connected on to robust edges.

\section{f) Histogram Equalization}

This could be a system for changing picture intensities to support qualification. This approach here and there will expand the overall differentiation of the numerous photos, especially once the usable data of the picture is depicted by shut refinement esteems. Through this modification, the forces might be higher appropriated on the histogram. This empowers for regions of lower local refinement to accomplish the following qualification. Histogram equalization achieves this by successfully spreading out the first incessant power esteems. The system is advantageous in pictures with foundations and closer views that are each brilliant or every dim.

A key advantage of the strategy is that it's a reasonably simple technique associated with an invertible operator.

\subsection{Segmentation Module}

Therefore, once the edge location and histogram equalization, the division of the picture is administrated utilizing accommodative locale developing. District developing could be a direct picture division procedure dependent on the area [19]. It's also delegated a pixel-based picture division approach since it includes the decision of introductory seed focuses. This way to deal with division analyzes the neighbor pixels of starting "seed focuses" and decides if the pixel neighbors should be added to the locale or not upheld certain conditions. The strategy is iterated to yield entirely unexpected areas. In an extremely conventional district developing method, the neighbor pixels are inspected by exclusively the "power" oblige. For this, a power level for force worth is prepared and those neighbor pixels that fulfill this edge is picked for district developing. The conventional district developing has the wince that clamor or variety of force could prompt openings or over-division. Another hindrance is that the methodology probably won't recognize the concealing of the significant pictures. For improving the typical district developing and adequately hooking the downsides of a conventional area developing, accommodative locale developing is anticipated. Regularly, an isolated space by district developing could contain little gaps. So as to maintain a strategic distance from this situation, the anticipated area developing refutes little openings inside the districts.

The district developing could be a three-advance technique that comprises of gridding, the decision of seed reason, applying locale developing to the reason. In gridding, one picture is part of numerous littler pictures by illustration a non-existent network over it. That is gridding winds up in changing the picture into numerous littler matrix pictures. The lattices are ordinarily square in structure and consequently, the network assortment to that the underlying picture is part into could be a variable. So as to finish a straightforward examination, the outcomes ought to be a little work arrange. Rising entryway district is shaped to choose the beginning stage for the underlying advance of the network. The underlying area of the seed is utilized as a 
particular area. At that point, the histogram examination of the seeds is utilized to decide the matrix point. The histogram for every pixel framework is found. As the picture is a grayscale picture, the picture estimations of 0 to every one of the lattice, histogram estimation of the most much of the time picked as the seed point pixel 255. Along these lines, the seed point pixel is taken as a beginning stage for any lattice. The power limit is the most extreme pixel estimation of the neighboring pixel worth might be characterized diversely under examination. Assume that a pixel force esteem Ip And neighbouring pixel esteem IN and the power is characterized as the limit TI after the IT then

\section{$\|\operatorname{Ip}-N\| \leq I$,}

Compressed to reach the intensity and satisfaction.

When the barrier satisfies the intensity of neighboring pixels region are grown and develop the territory. Then, the area has grown to be extracted for each grid and agricultural characteristics of the areas. After this process, the cancellation has to be verified, then it is left to small areas.

\subsection{Kalman Filtering Module}

The input image is Kalman filtered and compared with the comparison is administrated with the assistance of the OR operator and also the ensuing figure provides the free space. Kalman filtering [19] is standard that uses a progression of estimation ascertained over a period holding noise and different inaccuracies, and produces evaluations of obscure factors that tend to be a lot of precise than those supported one measure alone. The Kalman filter may be a repetitive assessor, this implies that solely the calculable state from the last time step and furthermore present measure are required to figure the approximate calculation for the present state. The Kalman filtering rule works during a two-step method within the vaticination. The Kalman filter engenders estimates of the present state factors, at the side of their uncertainties. Once the end result of ensuing measure (necessarily corrupted with some quantity of error, as well as random noise) is ascertained, these calculations are upgraded employing a weighted average, with a lot of weight being given to gauges with higher sureness.

The Kalman filter model [19] for the system and a specific given to estimates with higher certainty.

Time instant is given as shown below:

$$
Y_{i}=n_{i} Y_{i}+Z_{i}
$$

Where is $\mathrm{n} \times \mathrm{n}$ matrix and is the sequence vector and is the vector of $n$-dimension in random in the given system? There is n-dimensional vector obtainable which is tainted by a commotion at the time, and now the following articulation as beneath:

$$
K_{i}=R_{i} Y_{i}+N_{i}
$$

Where vector is the additive noise in the processing and is termed the $\mathrm{m} \times \mathrm{n}$ observation matrix. Assuming that the vectors, and are mutually related to one another, which results in the expression: metameric image to possess the free space calculation. The

$$
G\left[Z_{i} N_{i}^{J}\right]=\varphi, i=0,1, \ldots
$$

Where represents the null matrix. Grounded these reflections, Kalman filtering equation can be derived as below,

$$
Y_{i}=n_{i} Y_{i-1}+H_{i}\left[K_{i}-R_{i} Y_{i-1}\right]
$$

Let the input image be represented by and let the image after the Kalman filtering be represented by . Let the image after the segmentation be represented by . Here, the segmented image is compared with the Kalman filtered image as shown in figure 3:

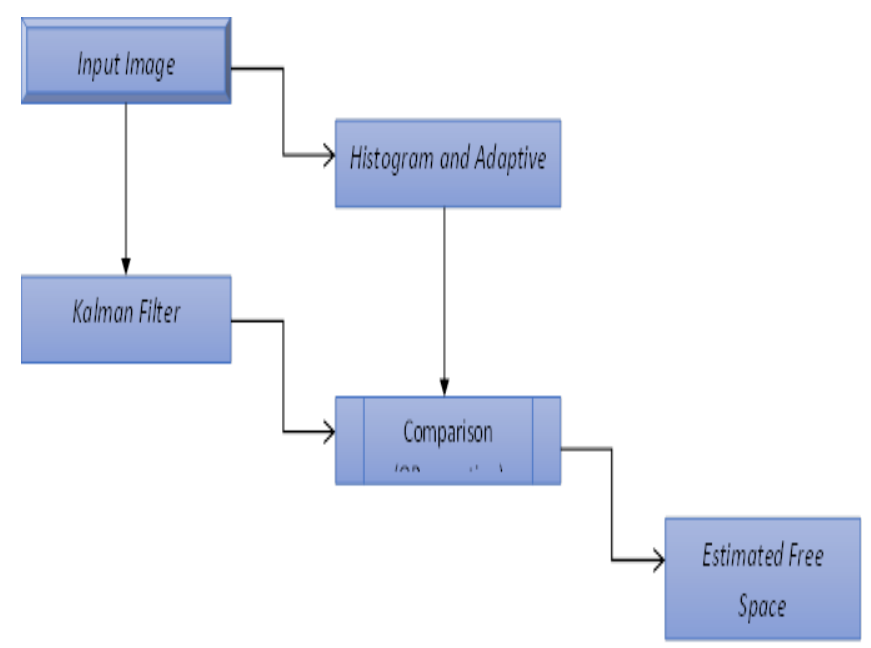

Figure 3: Kalman Filtering module

The comparison is carried out using the OR operator. The OR operator generally takes two pictures as input and outputs a third picture whose constituent values are simply those of the primary mage, ORed with the corresponding pixels from the second. In our case, the Kalman filtered image is ORed with a segmented picture to have the ORed image. The operation is performed straightforwardly in a single pass. It is essential that all the input pixel values being worked on have a consistent number of bits in them or abrupt things could happen where the pixel values within input pictures don't appear to be simple 1-bit numbers, the OR operation is commonly dispensed severally on each relating bit inside the pixel values, in bitwise manner. The ORed picture offers free space estimation.

\section{RESULTS AND DISCUSSIONS}

The proposed free space measurement method is considered with the help of experimental outcomes in this unit. In 5.1, the set up for the experiment and evaluation metrics are discussed. Simulation outcomes obtained are given in unit 4.2 and performance analysis is made in unit 5.3 . 


\subsection{Experimental Set Up and Evaluation Metrics}

The proposed procedure is executed utilizing MATLAB on a framework having the design of 6 GB RAM and 2.8 $\mathrm{GHz}$ Intel I-7 processor. The assessment measurements used to assess the proposed method comprises of affectability, explicitness, and exactness. So as to discover these measurements, we initially figure a portion of the conditions of True positive (TP), True negative (TN), False negative $(\mathrm{FN})$ and False positive (FP) in light of the definitions given in table 1.

Table 1: Table outlining the terms TP, FP, FN, and TN

\begin{tabular}{lll}
\hline Experimental Outcome & $\begin{array}{l}\text { Condition as outlined by the Definition } \\
\text { Standard of Truth }\end{array}$ \\
\hline Positive & Positive & True Positive (TP) \\
Positive & Negative & False Positive (FP) \\
Negative & Positive & False Negative (FN) \\
Negative & Negative & True Negative(TN)
\end{tabular}

The evaluation metrics of sensitivity, specificity, and accuracy can be expressed in terms of TP, FP, FN, and TN. Sensitivity is the proportion of true positives that are correctly identified by a diagnostic test. It shows how good the $\mathrm{t}$ The assessment measurements of affectability, explicitness, and precision can be communicated in terms of TP, FP, FN, and TN. Affectability is the extent of genuine positives that are effectively distinguished by a demonstrative test. It demonstrates how great the test is at distinguishing a malady. est is at detecting disease.

\section{Sensitivity $=\mathrm{TP} /(\mathrm{TP}+\mathrm{FN})$}

Specificity is the proportion of the true negatives correctly identified by a diagnostic test. Identifying norm Particularity is the extent of the genuine negatives accurately recognized by a demonstrative test. Recognizing typical (negative) condition to recommend how great it. al (negative) condition to suggest how good it.

$$
\text { Specificity }=T N /(T N+F P)
$$

Precision is the extent of genuine outcomes, either evident positive or genuine negative, in a populace. It quantifies the level of veracity of an analytic test on a condition.

$$
\text { Accuracy }=(T N+T P) /(T N+T P+F N+F P)
$$

\subsection{Simulation Results}

Here, the output of the simulation obtained for the proposed technique are given. Figure 4 gives the simulation results of the input image, floor marked input image, edge marked input image, edge detected using canny edge detector, clustered image using FCM, Kalman filtered image, clustered image and output image. In the output image, free space is marked in red.

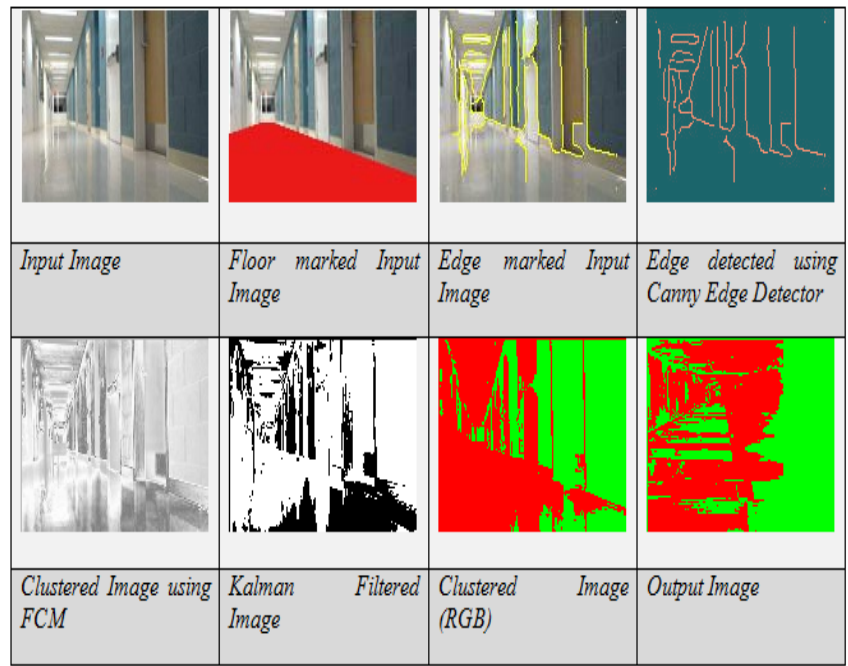

Figure 4: Simulation Results

\subsection{Performance Analysis}

the exhibition of the proposed procedure is investigated with the utilization of assessment measurements of affectability, particularity, and exactness.

Table 2: Evaluation metric values obtained for varying cluster sizes

\begin{tabular}{|c|c|c|c|}
\hline & \multicolumn{3}{|c|}{ Clusters } \\
\cline { 2 - 4 } & $\mathbf{2}$ & $\mathbf{3}$ & $\mathbf{4}$ \\
\hline True Positive & 0.90 & 0.63 & 0.85 \\
\hline True Negative & 0.50 & 0.50 & 0.50 \\
\hline False Positive & 0.50 & 0.50 & 0.50 \\
\hline False Negative & 0.10 & 0.37 & 0.15 \\
\hline Affectability & 0.90 & 0.63 & 0.85 \\
\hline particularity & 0.50 & 0.50 & 0.50 \\
\hline exactness & 0.71 & 0.61 & 0.68 \\
\hline
\end{tabular}

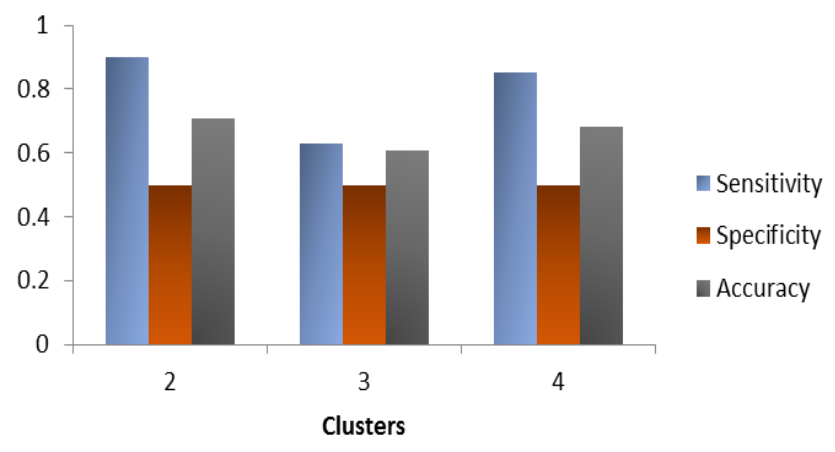

Figure 5: Chart of evaluation metric values obtained for varying cluster sizes 


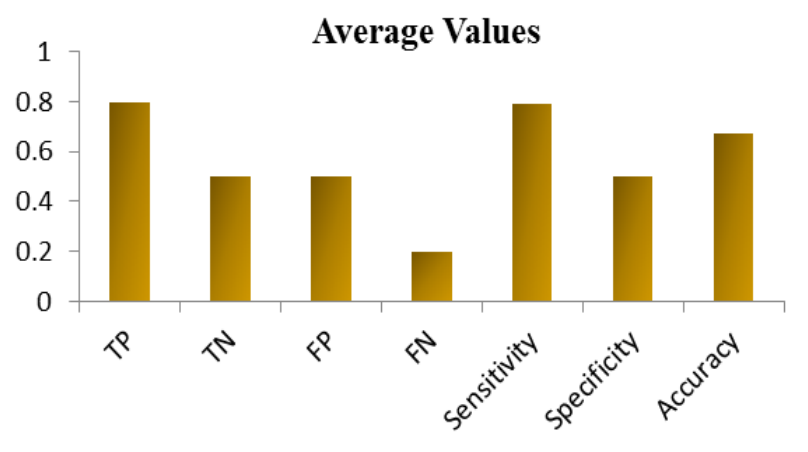

Figure 6: Chart of average evaluation metric values obtained

Inferences from figures 5, 6 and table 2:

- Figures 5, 6 and Table 2 gives the evaluation metrics obtained for the proposed technique.

- The evaluation in consideration includes FP, TP, FN, $\mathrm{TN}$, affectability, particularity and exactness.

- Figure 5 and Table 2, gives the evaluation metrics obtained by varying the cluster size. Cluster sizes in consideration are 2, 3 and 4 .

- It can be seen from the results that all cases have yielded good results. Amongst, the 00best results came when cluster size was taken for 2 .

- Highest affectability, particularity, and exactness came about $0.90,0.50$ and 0.71 respectively (when cluster size was taken as 2).

- Figure 6 gives the average obtained considering all cluster sizes.

- The average TP, TN, FP, and FN came about $0.79,0.5$, 0.5 and 0.20 respectively. The average affectability, particularity, and exactness came about $0.79,0.50$ and 0.67 respectively.

- The high average values indicate the good performance of the proposed technique in the area.

\section{CONCLUSION}

Free space measurement for a blind person using histogram equalization and adaptive region growing is projected in this paper. The method comprises of three segments, namely histogram equalization module, segmentation module, and Kalman filtering module. The proposed technique is evaluated under standard evaluation metrics of TP, FP, TN, FN, sensitivity, specificity and accuracy for varying cluster sizes of 2, 3 and 4 . The simulations results obtained are plotted. Highest affectability, particularity, and exactness came about $0.90,0.50$ and 0.71 and similarly, the average TP, TN, FP, and FN came about $0.79,0.5,0.5$ and 0.20 respectively. Analyzing the cluster performance, the cluster size of 2 gave the best results. The high evaluation metric values of sensitivity, specificity, and accuracy indicate the good performance of the proposed technique in the area.

\section{ACKNOWLEDGEMENTS}

The manuscript is prepared by taking assistance from Accendere Knowledge Management Services Pvt. Ltd, we are thankful to them. We also express our gratitude to our teachers and mentors for guiding us throughout the work.

\section{REFERENCES}

1. A. Dhanshri, K. R. Kashwan, "Identification Of Dynamic Objects Using Matlab And Labview For Aiding Blind Person", International Conference on Electronics and Communication Engineering,pp.142-149, 2013.

2. João José, Miguel Farrajota, João M.F. Rodrigues and J.M. Hans du Buf, "The Smart Vision Local Navigation Aid for Blind and Visually Impaired Persons", International Journal of Digital Content Technology and its Applications, Vol.5 No.5, pp.362-374,2011.

3. S. Nithya and A. S. L Shravani, "Electronic Eye for Visually Impaired Persons", International Journal of Emerging Technology and Advanced Engineering, Vol. 3, No. 10, pp.700-704, 2013.

4. Lorenzo Picinali, Amandine Afonso1, Michel Denis and Brian F.G. Katz, "Exploration of architectural spaces by blind people using auditory virtual reality for the construction of spatial knowledge", International Journal of Human-Computer Studies, Vol. 72, No.4, pp.393-407, 2014.

5. O.D.Faugeras.Three - Dimensional Computer Vision: a Geometric Viewpoint.MIT Press, 1993.

6. O.D.Faugeras.Stratification of three-dimensional vision: Projective, affine, and metric representation, Journal of the Optical Society of America, A12:465-484, 1995.

7. E.H.Elsayed, T.Ahmed Monumentally A.Farag, TheCardEye: A Trinocular Active Vision System, Springer Berlin/Heidelberg, Vol 2095/2001.

8. Z.Zhang," Flexible Camera Calibration by Viewing a Plane from Unknown Orientation", ICCV '97, 1997.

9. Avinash N., Murali S.," Camera Center Estimation Using Vanishing Points", IEEE 1st International Conference on Signal and Image Processing.2006., Volume1, On page(s):467, 2006.

10. Yinxiao $\mathrm{Li}$ and Stanley T. Birchfield, "Image-Based Segmentation of Indoor Corridor Floors for a Mobile Robot", IEEE International Conference on Intelligent Robots and Systems (IROS), pp.837 - 843, 2010.

11. BhuvaneshArasu and SenthilKumaran, "Blind Man's Artificial EYE An Innovative Idea to Help the Blind", International Journal of Engineering Development and Research, pp.205-208,2014.

12. Vincent Gaudissart, Silvio Ferreira, Céline Thillou, Bernard Gosselin, "Mobile Reading Assistant for Blind People", Conference Speech and Computer, pp. 538-544, 2004.

13. H. Fernandes, N. Conceição, H. Paredes, A. Pereira, P. Araújo and J. Barroso, "Providing accessibility to blind people using GIS", Universal Access in the Information Society, Vol. 11, No. 4, pp 399-407, 2012.

14. Anke M. Brocka, Philippe Truilleta, Bernard Oriolaa, DelphinePicardb and Christophe Jouffraisa, "Interactivity Improves Usability of Geographic Maps for Visually Impaired People", Human-Computer Interaction, Vol. 30, No. 2, pp.156-194, 2015.

15. S. Kammouna, G. Parseihianc, O. Gutierreza, A Brilhaulta, A. Serpaa, M. Raynala, B. Oriolaa, M.J.-M. Macéa, M. Auvrayc, M. Denisc, S.J. Thorpeb, P. Truilleta, B.F.G. Katzc and C. Jouffraisa, "Navigation and space perception assistance for the visually impaired: The NAVIG project", IRBM, Vol. 33, No. 2,pp.182-189, 2012. 
16. Wersényi and György, "Virtual Localization by Blind Persons", JAES Vol.60, No.7/8, pp. 568-579, 2012.

17. Sergei Azernikov. Sweeping solids on manifolds. In Symposium on Solid and Physical Modeling, pages 249-255, 2008.

18. F. Mai, Y. Hung, H. Zhong, and W. Sze. A hierarchical approach for fast and robust ellipse

19. extraction. Pattern Recognition, 41(8):2512-2524, August 2008.

20. S. J. Julier and J. K. Uhlmann, "Unscented filtering and nonlinear estimation," Proc. IEEE, vol. 92, no. 3, pp. 401-422, 2004.

21. W. K. Pratt, Digital Image Processing 4th Edition, John Wiley \& Sons, Inc., Los Altos, California, 2007. 